\title{
Incidence and etiology of omphalitis in Pakistan: a community-based cohort study
}

\author{
Fatima Mir ${ }^{1}$, Shiyam Sundar Tikmani ${ }^{1}$, Sadia Shakoor ${ }^{2}$, Haider Javed Warraich ${ }^{1}$, Shazia \\ Sultana ${ }^{1}$, Syed Asad Ali ${ }^{1}$, Anita K M Zaidi ${ }^{1,2}$ \\ ${ }^{1}$ Department of Pediatrics and Child Health, Aga Khan University, Karachi, Pakistan \\ ${ }^{2}$ Division of Microbiology, Department of Pathology, Aga Khan University, Karachi, Pakistan
}

\begin{abstract}
Introduction: Although omphalitis (umbilical infections) among newborns is common and a major cause of neonatal deaths in developing countries, information on its burden and etiology from community settings is lacking. This study aimed to determine the incidence and etiology of omphalitis in newborns in high neonatal mortality settings in Karachi, Pakistan.

Methodology: Trained community health workers surveyed all new births in three low-income areas from September 2004 to August 2007. Pus samples from the umbilical stumps were obtained from babies with pre-defined signs of illness and subjected to culture and antimicrobial susceptibility testing.

Results: Among 6904 births, 1501 (21.7\%) newborns were diagnosed with omphalitis. Of these, 325 (21.6\%) were classified as mild, 1042 $(69.4 \%)$ as moderate, and $134(8.9 \%)$ as severe; $141(9.3 \%)$ were associated with clinical signs of sepsis. The incidence of omphalitis was 217.4/1000 live births; moderate-severe omphalitis 170.3 per 1000 live births; and associated with sepsis 20.4 per 1000 live births. Of 853 infants with purulent umbilical discharge, $64 \%$ yielded 583 isolates. The most common pathogens were Staphylococcus aureus, of which 291 (95.7\%) were methicillin-susceptible Staphylococcus aureus (MSSA) and 13 (4.2\%) methicillin-resistant S. aureus (MRSA); Streptococcus pyogenes 105 (18\%); Group B beta-hemolytic streptococci 59 (10\%); Pseudomonas spp., 52 (8.9 \%); Aeromonas spp. 19 (3.2\%); and Klebsiella spp. $12(2 \%)$.

Conclusions: A high burden of omphalitis can be associated with sepsis among newborns in low-income communities in Pakistan. S. aureus is the most common pathogen isolated from umbilical pus. Appropriate low-cost prevention strategies need to be implemented.
\end{abstract}

Key words: incidence; etiology; omphalitis; community acquired; umbilical infection; Staphylococcus aureus; MRSA; newborn; antimicrobial susceptibility

J Infect Dev Ctries 2011; 5(12):828-833.

(Received 24 May 2010 - Accepted 21 April 2011)

Copyright $(0) 2011$ Mir et al. This is an open-access article distributed under the Creative Commons Attribution License, which permits unrestricted use, distribution, and reproduction in any medium, provided the original work is properly cited.

\section{Introduction}

Neonatal infections account for $12 \%$ of global child mortality [1]. In developing countries such as Pakistan, inadequate attention to skilled birth attendance has led to half of all child deaths occurring in the neonatal period, with sepsis as one of the major causes of death [1]. Umbilical infections (omphalitis) are common among newborns in developing countries and may predispose to lifethreatening neonatal sepsis [2-4]. Incidence rates in newborns in nurseries from developing countries range from 2 per 1000 to 54 per 1000, with figures from Turkey as high as 77 per 1000 live births. Case fatality rates range from $0-15 \%$ in these hospitalized. Very few population-based studies on rates of umbilical infection have been reported [5-8]. Predictably, community-based rates for omphalitis are much higher (105 per 1000 live births in Nepal) due to the co-existence of many risk factors such as the following the high proportion of babies born at home; low rates of peri-partum skilled birth attendants; sub-optimal use of infection control practices during and after birth (hand washing, disinfection of delivery surface and instruments, sterile cord cutting and tie methods); cultural practices involving application of unsafe substances such as cow dung to the cord; and delayed healthcare seeking behavior among families [5]. These suboptimal peri-partum practices are prevalent in South Asia and may predispose to as high if not higher incidence rates of omphalitis in countries such as Pakistan $[9,10]$.

The objective of this study was to determine the incidence and etiology of non-tetanus omphalitis in low-income community settings of Karachi, where neonatal mortality is high and home births 
Table 1. Grading of omphalitis on the basis of severity

Mild
Redness extending to umbilical base but $<2 \mathrm{~cm}$ of abdominal wall around umbilical stump involved
No associated purulent discharge
Moderate
Redness around umbilical stump $<2 \mathrm{~cm}$ with associated purulent discharge, or purulent discharge alone
without any redness
Severe
Redness around umbilical stump with $>2 \mathrm{~cm}$ extension to abdominal wall, and/or swelling around
umbilicus
With or without purulent discharge

predominate. We identified the most common causative organisms and their antimicrobial susceptibility patterns in a community cohort, with a secondary objective to observe association between omphalitis and sepsis and establish appropriate management strategies for omphalitis.

\section{Methodology}

Study setting

Pakistan is a large developing country with an estimated population of 180 million. The neonatal mortality rate in Pakistan is 54 per 1000 live births, with two-thirds of all births occurring at home [7].

Karachi is Pakistan's largest city with about 18 million people. This study was conducted in three low-income (two peri-urban and one urban) communities in Karachi where newborn and young infant surveillance and care systems were established to conduct studies on clinical signs predictive of serious illness in young infants [8]. The primary source of income in the two peri-urban coastal areas is derived from fishing while in urban areas manual labor and employment in small factories prevail. Family earnings range from minimum wage to lowermiddle income, with the majority averaging less than $\$ 1.25$ per day (World Bank definition of extreme poverty). Baseline demographic data (from demographic surveillance conducted by the Department of Pediatrics and Child Health, Aga Khan University, in study sites) shows that neonatal mortality in the three study sites is 45 per 1000 . Most deliveries are conducted at home by traditional (unskilled) birth attendants (TBAs) who have no formal certification or licensure. The TBAs are local women, often illiterate, who provide pregnancy and child birth care based primarily on experience and knowledge acquired informally through the traditions and practices of the communities. In addition, the use of sterile delivery kits (as an indicator of effective infection control practices) in home deliveries is low $(32 \%)[6,7,9]$.

Each community's primary health-care needs for children are provided by Aga Khan University (AKU) Department of Pediatrics and run by the Primary Health Care (PHC) center, which is staffed with physicians and community health workers.

The appropriate approval was obtained from the Aga Khan University's Ethical Review Committee.

\section{Study design}

Community health workers (CHWs) were trained to detect clinical signs of serious illness in enrolled pregnant women and newborns through periodic household surveillance in the three sites. Newborn visits by CHWs were conducted within 48 hours of birth and after one week, two weeks, and one month of birth. Over $70 \%$ of newborns born at home were seen within 48 hours of birth because of strong links established with local TBAs. Newborns with omphalitis or other illnesses were referred to the PHC center where they were evaluated by study physicians.

For CHW training purposes, a standard picture set was developed by the principal investigator that showed various clinical presentations of omphalitis. The diagnosis of omphalitis was made following the criteria indicated in Tables 1 and 2. If redness extending to the base of the umbilical stump or surrounding abdominal wall was present, and/or purulent discharge from the umbilical stump was noted, the patient was diagnosed with omphalitis. These infections were categorized as mild, moderate, or severe according to definitions based on clinical algorithms from prior community-based work (Table 1) [10]. In addition, babies were also examined for systemic signs of sepsis (Table 2). 
Table 2. Clinical Definition of Sepsis

\begin{tabular}{|l|l|}
\hline \multicolumn{2}{|l|}{ Any three of the following: } \\
\hline 1 & Respiratory Rate $>60 /$ minute \\
\hline 2 & Feeding difficulty/weak suck \\
\hline 3 & Fever $>37.5^{\circ} \mathrm{C}$ (axillary) \\
\hline 4 & Temperature $<36.0^{\circ} \mathrm{C}$ (axillary) and not increasing on warming \\
\hline 5 & Lethargic or $<$ normal movement \\
\hline 6 & Excessive crying or irritability \\
\hline 7 & Weak, abnormal or absent cry \\
\hline 8 & Persistent vomiting (last 3 feeds) \\
\hline 9 & Abdominal distension \\
\hline 10 & Hypoglycemia - blood glucose $<40 \mathrm{mg} / \mathrm{dl}$ \\
\hline 11 & Presence of skin, eye, or local umbilical infection \\
\hline
\end{tabular}

\section{Sample collection and specimen processing}

Swabs in Amie's medium (Medical Wire and Equipment, Wiltshire, UK) used to culture babies with umbilical purulent discharge were transported to the Aga Khan University Hospital (AKUH) Clinical Microbiology Laboratory within five hours of collection. Swabs were plated directly to locally prepared 5\% sheep blood agar, chocolate agar and MacConkey agar plates. After aerobic incubation of MacConkey plates $\left(35 \pm 2^{\circ} \mathrm{C}\right.$, air) and chocolate and sheep blood agars $\left(5-10 \% \mathrm{CO}_{2}\right)$ for 24 and 48 hours, the results were reported semi-quantitatively as few colonies, moderate growth, or heavy growth of the recovered organism. Gram-negative bacteria were identified by API 20E and API 20NE kits (bioMérieux, Marcy l'Etoile, France) and S. aureus was identified by the tube coagulase test. Lancefield groups of streptococci were determined by latex agglutination with appropriate anti-sera using the PathoDx kit (Remel Inc., Thermo Fisher Scientific USA).

All antimicrobial susceptibilities were performed by the Kirby-Bauer disk diffusion method following the guidelines established by the Clinical Laboratory Standards Institute [11]. The D-test to detect the macrolide-lincosamide-streptogramin-B inducible (MLSBi) phenotype was determined for isolates of $S$. aureus, S. pyogenes, and $S$. agalactiae.

Blood cultures were collected from 56 of 141 (39.7\%) infants with suspected sepsis and omphalitis whose parents provided consent. After skin disinfection with $70 \%$ alcohol swabs, blood was collected in a BACTEC Peds Plus/F bottle (Becton Dickinson, Franklin Lakes, NJ, USA) and transported to the Aga Khan University Clinical Microbiology Laboratory for detection in the automated BACTEC 9240 instrument (Becton Dickinson, Franklin Lakes, NJ, USA). When flagged positive by the instrument, the bottles were examined by Gram stain and bacteria were identified by tests mentioned previously for umbilical cultures [12].

\section{Data analysis}

Data was analyzed by the Data Management Unit, Department of Pediatrics, Aga Khan University. Incidence rates and confidence intervals were calculated using SPSS16.0 (SPSS, Chicago, IL, USA). The primary outcomes of omphalitis determined from predominantly home-delivered babies in the community were incidence, etiology, and antimicrobial susceptibilities of the common pathogens causing infections.

\section{Results}

Incidence

During the study period of September 2004 to August 2007, there were a total of 6904 births in the three surveillance areas, $65 \%$ of which were delivered by TBAs. Among the birth cohort, 1501 $(21.7 \%)$ were diagnosed with omphalitis by the CHWs. Of these, physicians categorized 325 (21.6\%) as mild, $1042(69.4 \%)$ as moderate, and $134(8.9 \%)$ 
as severe infections. The incidence of omphalitis was 217.4/1000 live births (95\% CI $=207.8-227.3)$; moderate to severe omphalitis, $170.3 / 1000$ live births $(95 \% \mathrm{CI}=160.8-180.3)$ and the incidence of omphalitis with sepsis was $20.4 / 1000$ live births $(141 / 6904)(95 \% \mathrm{CI}=17.3-24)$. With home or clinic-based antimicrobial therapy, case fatality rate for omphalitis alone was $0.15 \%(2 / 1360)$ and for omphalitis with sepsis, it was $0.7 \%$ by day $7(1 / 141)$. The overall case fatality observed in this cohort of infants with omphalitis was 3/1501 (0.2\%).

\section{Etiology}

Cultures were collected and processed in 675 (79\%) from a total of 853 babies with umbilical purulent secretions. Of 675 specimens cultured, 432 (64\%) yielded 583 pathogens; 299 (69\%) were pure cultures and $133(30.7 \%)$ were polymicrobial. Among the 141 babies with omphalitis and sepsis, 56 (40\%) received parental consent to have blood cultures drawn. However, only one blood culture grew $S$. aureus and one was polymicrobial (Klebsiella pneumoniae, E. coli, and Enterobacter spp).

Bacteria isolated from the umbilical exudate in descending order of frequency were as follows: $S$. aureus (52\%); Streptococcus pyogenes (Group A beta-hemolytic streptococci) (18\%); Streptococcus agalactiae (Group B beta-hemolytic streptococci) (10\%); Pseudomonas spp. (9\%); Aeromonas spp. (3.2\%); and Klebsiella spp. (2\%). Umbilical cultures obtained from 54 of 141 babies (38\%) with omphalitis and sepsis grew pure cultures in 19 and polymicrobial cultures from 13 cases. Gram-negative bacteria were isolated from $17.8 \%$ (8/45) of umbilical cultures obtained from patients with omphalitis and sepsis and 17\% (99/583) from those with moderatesevere omphalitis irrespective of sepsis.

\section{Antimicrobial susceptibility patterns}

Among 304 S. aureus isolates, 13 (4.3\%) were methicillin-resistant (MRSA), 291 (95.7\%) were methicillin-susceptible (MSSA), 240 (78.9\%) were erythromycin- susceptible, $287 \quad(94.4 \%)$ were clindamycin-susceptible, and $204(67.1 \%)$ were cotrimoxazole-susceptible. Two percent of all $S$. aureus were $\mathrm{D}$-test positive. From 164 betahemolytic streptococci (Group A $=105$, Group B = 59), $121(73.7 \%)$ were erythromycin-susceptible (Group $\mathrm{A}=77$, Group B = 44), and $142(86.5 \%)$ were clindamycin-susceptible (Group A = 91, Group
$\mathrm{B}=51)$. Fifty percent of $S$. pyogenes was D-test positive and all $S$. agalactiae were D-test negative.

Results for commonly isolated Gram-negative bacteria showed $100 \%$ susceptibility to gentamicin and amikacin with Pseudomonas spp. $(\mathrm{n}=52)$, Aeromonas spp. $(\mathrm{n}=19)$, and E. coli $(\mathrm{n}=9)$; and $92 \%$ with Klebsiella spp. $(\mathrm{n}=12)$. Ciprofloxacin susceptibility was $100 \%, 98 \%, 91.6 \%$ and $89 \%$ with Aeromonas spp., Pseudomonas spp., Klebsiella spp. and $E$. coli, respectively. Susceptibility to ceftriaxone was $91.6 \%$ with Klebsiella spp. and $89 \%$ with E.coli.

\section{Discussion}

The burden of omphalitis in our population was determined to be 217.4/1000 live births, a rate comparable to that of Gadchiroli, rural Maharashtra, India (197/1000 live births) [13]. We provide additional information regarding the etiology and antimicrobial susceptibility patterns of pathogens causing omphalitis in the study patients.

The umbilical cultures obtained from our patients grew predominantly Gram-positive bacteria, in contrast to one hospital-based study from India where Gram-negative bacteria predominated [14]. This may reflect higher colonization rates with Gram-negative bacteria in babies born in hospitals compared to those born at home. Results demonstrated that $S$. aureus and beta-hemolytic streptococci accounted for $80 \%$ of all pathogens causing community-acquired omphalitis. The predominance of $S$. aureus suggests the inclusion of an anti-staphylococcal agent (e.g., cloxacillin) in treatment regimens for infants with omphalitis either with or without sepsis. Although an overall low incidence of MRSA (umbilical cultures) from the community was observed, most of the cases were recovered in the latter half of the study period, which may be indicative of a rise in communityacquired MRSA infections.

It is noteworthy that Group B streptococci (GBS) were isolated from $10 \%$ of umbilical exudates but none from blood cultures. The low positivity rate of GBS in blood cultures has also been reported in other developing areas (range of $0.22 \%$ in East Asia/Pacific to $15 \%$ in Africa). A culture positivity rate of $7 \%$ in South Asia stems from community surveillance in Karachi, Pakistan, and tertiary center data in Sevagram and Karnataka, India [15]. GBS neonatal sepsis may be under-detected in South Asia due to the inability to obtain blood cultures within the first 48 hours of life when these bloodstream infections most commonly occur. Newborns delivered at home who develop sepsis do not usually 
present to medical facilities to obtain blood cultures and hospital-born babies are often treated with antimicrobial agents before obtaining cultures [16].

Among GBS and $S$. pyogenes isolates, resistance to macrolides (approximately 25\%) was higher while that to clindamycin (approximately 13\%) was similar to hospital cohort data from India $(17.6 \% S$. pyogenes; $14.3 \%$ GBS) [29-30]. Since macrolides are commonly used to treat respiratory infections in Pakistan, the drug resistance may be a consequence of the availability of antibiotics over the counter without the need of a doctor's prescription.

Reassuringly, antimicrobial resistance in enteric Gram-negative bacteria and Pseudomonas spp. was found to be quite low. This is in contrast to the high number of extended spectrum beta-lactamase (ESBL) producing E.coli and other enteric pathogens reported from urinary tract infections in developing countries [17-24]. Of interest, Aeromonas spp., a known enteropathogen causing diarrhoea, was shown to colonize and possibly infect newborn babies in our study. The clinical consequences of this are unknown.

One limitation of this study was the difficulty in distinguishing the routine physiological inflammatory response and redness associated with cord separation from infected umbilical stumps in cases where discharge is not present. Thus some of the 325 cases (22\%) classified as mild omphalitis (redness without discharge) may have represented physiological redness only. Thus we may have over-estimated the true burden of omphalitis in this population. However, even if these cases are excluded, the estimated incidence of omphalitis would be 170 cases per 1000 live births. It is highly recommended that standardized and validated diagnostic criteria defining omphalitis should be developed by the World Health Organization to enable accurate recognition and treatment.

This study did not evaluate possible risk factors contributing to the high incidence of omphalitis in the study population, notably low birth weight and inadequate birth attendant and maternal education. As previously reported, omphalitis could result from the practice of applying non sterile products to the umbilical stump, such as mustard or coconut oil, surma (antimony or lead containing compound used to line eyelids), soil or ghee (home-made unclarified butter), or animal dung. In addition, unsanitary delivery practices, including poor hand washing, unclean delivery surfaces, and unsterile cord cutting and tying are other contributory factors to developing omphalitis $\quad[3,5,25]$. Simple, cost-effective interventions can be implemented to reduce risks associated with omphalitis [4,5,9]. Although untreated omphalitis is a high mortality condition [2], the overall case fatality rate of omphalitis in our study was low $(0.2 \%, 3 / 1501)$. This was most likely a consequence of early recognition through active surveillance and institution of appropriate antimicrobial therapy in a research setting. Reported case fatality rates in hospital cohorts have ranged from $13-19 \%$ [14,26]. In a community-based cohort in rural Nepal, the risk of mortality was found to be $46 \%$ higher (95\% CI: 8-98\%) among infants with omphalitis compared with those without [2]. Use of chlorhexidene antiseptic on the umbilical stump for prevention of omphalitis in this trial reduced neonatal mortality by $24 \%$ (RR 0.76 , 95\% CI $0.55-1.04$ ) compared to dry cord care [4]. Implementation of preventive strategies, as well as early and accurate diagnosis, and appropriate therapeutic management for newborns with omphalitis with and without sepsis are urgently needed for developing countries $[25,27,28]$.

\section{Acknowledgements}

The authors would like to thank Ms Aatekah Owais and Dr Farah Naz Qamar for help with data analysis. Editorial help provided by Shamsa Panjwani is also gratefully acknowledged.

Dr. Fatima Mir and Dr. Shiyam Sunder received research training support from the National Institute of Health's Fogarty International Center (1 D43 TW007585-01). The parent studies during which these data were collected were supported by the Saving Newborn Lives Initiative at Save the Children, USA, funded by the Bill and Melinda Gates Foundation.

\section{References}

1. Black RE, Cousens S, Johnson HL, Lawn JE, Rudan I, Bassani DG, Jha P, Campbell H, Walker CF, Cibulskis R (2008) Global, regional, and national causes of child mortality in 2008: a systematic analysis. The Lancet 375: 1960-1987.

2. Mullany LC, Darmstadt GL, Katz J, Khatry SK, LeClerq SC, Adhikari RK, Tielsch JM (2009) Risk of mortality subsequent to umbilical cord infection among newborns of southern Nepal: cord infection and mortality. Pediatr Infect Dis J 28: 17.

3. Mullany LC, Darmstadt GL, Katz J, Khatry SK, LeClerq SC, Adhikari RK, Tielsch JM (2007) Risk factors for umbilical cord infection among newborns of southern Nepal. Am J Epidemiol 165): 203.

4. Mullany LC, Darmstadt GL, Khatry SK, Katz J, LeClerq SC, Shrestha S, Adhikari R, Tielsch JM (2006) Topical applications of chlorhexidine to the umbilical cord for prevention of omphalitis and neonatal mortality in southern Nepal: a community-based, cluster-randomised trial. The Lancet 367: 910-918. 
5. Fikree FF, Ali TS, Durocher JM, Rahbar MH (2005) Newborn care practices in low socioeconomic settlements of Karachi, Pakistan. Soc Sci Med 60: 911-921

6. Ariff S, Soofi SB, Sadiq K, Feroze AB, Khan S, Jafarey SN, Ali N, Bhutta, ZA (2010) Evaluation of health workforce competence in maternal and neonatal issues in public health sector of Pakistan: an assessment of their training needs. BMC Health Services Research 10: 319.

7. National Institute of Population Studies (NIPS) [Pakistan], and Macro International Inc (2008) Pakistan Demographic and Health Survey, 2006-07. Islamabad, Pakistan: National Institute of Population Studies and Macro International Inc.

8. The Young Infants Clinical Signs Study Group (2008) Clinical signs that predict severe illness in children under age 2 months: a multicentre study. The Lancet 371: 135-42.

9. Darmstadt GL, Hassan M, Balsara ZP, Winch P J, Gipson R, Santosham M (2009) Impact of clean delivery-kit use on newborn umbilical cord and maternal puerperal infections in Egypt. J Health Popul Nutr 27: 746.

10. Mullany LC, Darmstadt GL, Katz J, Khatry SK, LeClerq SC, Adhikari RK, Tielsch JM (2006) Development of clinical sign based algorithms for community based assessment of omphalitis. Arch Dis Child 91: F99.

11. Clinical Laboratory Standards Institute (2006) Performance Standards for Antimicrobial Susceptibility Testing; Sixteenth Informational Supplement. Pennsylvania.

12. Isenberg HD (2004) Clinical microbiology procedures handbook, $2^{\text {nd }}$ edition. Washington, DC: ASM Press $2298 \mathrm{p}$.

13. Bang AT, Reddy HM, Bang RA, Deshmukh MD (2005) Why do neonates die in rural Gadchiroli, India? (Part II): estimating population attributable risks and contribution of multiple morbidities for identifying a strategy to prevent deaths. J Perinatol 25: S35-S43.

14. Faridi MM, Rattan A, Ahmad SH (1993) Omphalitis neonatorum. J Indian Med Assoc 91: 283.

15. Zaidi AKM, Thaver D, Ali SA, Khan TA (2009) Pathogens associated with sepsis in newborns and young infants in developing countries. Pediatr Infect Dis J 28: S10.

16. Global GBS Vaccine Working Group (2011) Group B streptococcal vaccine for resource-poor countries. The Lancet. In press.

17. Thaver D, Ali SA, Zaidi AKM (2009) Antimicrobial resistance among neonatal pathogens in developing countries. Pediatr Infect Dis J 28: S19.

18. Randrianirina F, Soares JL, Carod JF, Ratsima E, Thonnier V, Combe P, Grosjean P, Talarmin A (2006) Antimicrobial resistance among uropathogens that cause communityacquired urinary tract infections in Antananarivo, Madagascar. J Antimicrob Chemother. Br Soc Antimicrob Chemo 59: 309

19. Kurutepe S, Surucuoglu S, Sezgin C, Gazi H, Gulay M, Ozbakkaloglu B (2005) Increasing antimicrobial resistance in Escherichia coli isolates from community-acquired urinary tract infections during 1998-2003 in Manisa, Turkey. Jpn J Infect Dis 58: 159-161.

20. Kothari A and Sagar V (2008) Antibiotic resistance in pathogens causing community-acquired urinary tract infections in India: a multicenter study. J Infect Dev Ctries 2: 354-358.

21. Guidoni EBM, Berezin EN, Nigro S, Santiago NA, Benini V, Toporovski J (2008) Antibiotic resistance patterns of pediatric community-acquired urinary infections. Brazil J Infect Dis 12:3

22. Kariuki S, Revathi G, Corkill J, Kiiru J, Mwituria J, Mirza N, Hart CA (2007) Escherichia coli from communityacquired urinary tract infections resistant to fluoroquinolones and extended-spectrum beta-lactams. J Infect Dev Ctries 1: 257-262

23. Orrett FA, Davis GK (2006) A comparison of antimicrobial susceptibility profile of urinary pathogens for the years, 1999 and 2003. West Indian Med J 55: 95.

24. Ruppé E, Woerther PL, Diop A, Sene AM, Da Costa A, Arlet G, Andremont A, Rouveix B (2009) Carriage of CTXM-15-producing Escherichia coli isolates among children living in a remote village in Senegal. Antimicrob Agents Chemother 53: 3135 .

25. Bahl R, Martines J, Ali N, Bhan MK, Carlo W, Chan KY, Darmstadt GL, Hamer DH, Lawn JE, McMillan DD (2009) Research priorities to reduce global mortality from newborn infections by 2015. Pediatr Infect Dis J 28: S43.

26. Guvenc H,Aygiin AD, Yasar F, Soylu F, Guvenc M, Kocabay K (1997) Omphalitis in Term and Preterm Appropriate for Gestational Age and Small for Gestational Age Infants. J Trop Pediatr 43: 368-372.27. Bhutta ZA, Zaidi AKM, Thaver D, Humayun Q, Ali S, Darmstadt GL (2009) Management of newborn infections in primary care settings: a review of the evidence and implications for policy? Pediatr Infect Dis J 28: S22.

28. Darmstadt GL, Bhutta ZA, Cousens S, Adam T, Walker N, de Bernis L (2005) Evidence-based, cost-effective interventions: how many newborn babies can we save? The Lancet 365: 977-988.

29. Capoor MR, Nair D, Deb M, Batra K, Aggarwal P (2006) Resistance to erythromycin and rising penicillin MIC in Streptococcus pyogenes in India. Jpn J Infect Dis 59: 334336.

30. Sharmila V, Joseph NM, Babu TA, Chaturvedula L, Sistla S (2011) Genital tract group B streptococcal colonization in pregnant women: a South Indian perspective. J Infect Dev Ctries 5: 592-595.

\section{Corresponding author}

Dr Anita K. M. Zaidi

Professor, Department of Pediatrics and Child Health

Aga Khan University

Stadium Road PO Box 3500

Karachi 74800, Pakistan

Fax: (92 21) 493-4294, 493-2095

Telephone: (92 21) 4930051 Ext 4955, Direct: 486-4955

Email: anita.zaidi@aku.edu

Conflict of interests: No conflict of interests is declared. 